\title{
Detection of prognostic methylation markers by methylC- capture sequencing in acute myeloid leukemia
}

\author{
Yan Li ${ }^{1,2, *}$, Hongmei Zhao ${ }^{3, *}$, Qingyu $\mathrm{Xu}^{1,4, *}, \mathrm{Na} \mathrm{Lv}^{1,5}, \mathrm{Yu} \mathrm{Jing}^{1}$, Lili Wang ${ }^{1}$, Xiaowen \\ Wang $^{3}$, Jing Guo ${ }^{3}$, Lei Zhou ${ }^{1}$, Jing Liu ${ }^{1}$, Guofeng Chen ${ }^{1,4}$, Chongjian Chen ${ }^{3}$, Yonghui \\ $\mathbf{L i}^{1}$ and Li $\mathbf{Y} \mathbf{u}^{1,5}$ \\ ${ }^{1}$ Department of Hematology and BMT Center, Chinese PLA General Hospital, Beijing 100853, China \\ ${ }^{2}$ Department of Hematology, Hainan Branch of Chinese PLA General Hospital, Sanya 572013, China \\ ${ }^{3}$ Annoroad Gene Technology Co. Ltd., Beijing 100176, China \\ ${ }^{4}$ Medical School of Nankai University, Tianjin 300071, China \\ ${ }^{5}$ Department of Hematology, General Hospital of Shenzhen University, Shenzhen 518060, China \\ *These authors have contributed equally to this work \\ Correspondence to: Li Yu, email: liyu301@vip.163.com \\ Keywords: acute myeloid leukemia; next generation sequencing; MCC-Seq; DNA methylation; prognostic markers \\ Received: July 19, $2017 \quad$ Accepted: November 15, $2017 \quad$ Published: November 30, 2017 \\ Copyright: Li et al. This is an open-access article distributed under the terms of the Creative Commons Attribution License 3.0 \\ (CC BY 3.0), which permits unrestricted use, distribution, and reproduction in any medium, provided the original author and source \\ are credited.
}

\section{ABSTRACT}

Clinical and genetic features incompletely predict outcome in acute myeloid leukemia (AML). The value of clinical methylation assays for prognostic markers has not been extensively explored. We assess the prognostic implications of methylC-capture sequencing (MCC-Seq) in patients with de novo AML by integrating DNA methylation and genetic risk stratification. MCC-Seq assessed DNA methylation level in 44 samples. The differentially methylated regions associated with prognostic genetic information were identified. The selected prognostic DNA methylation markers were independently validated in two sets. MCC-Seq exhibited good performance in AML patients. A panel of 12 differentially methylated genes was identified with promoter hyper-differentially methylated regions associated with the outcome. Compared with a low M-value, a high $M-v a l u e$ was associated with failure to achieve complete remission $(p=0.024)$, increased hazard for disease-free survival in the study set $(p=0.039)$ and poor overall survival in The Cancer Genome Atlas set $(p=0.038)$. Hematopoietic stem cell transplantation and survival outcomes were not adversely affected by a high $M$-value $(p=0.271)$. Our study establishes that MCC-Seq is a stable, reproducible, and cost-effective methylation assay in AML. A 12-gene M-value encompassing epigenetic and genetic prognostic information represented a valid prognostic marker for patients with AML.

\section{INTRODUCTION}

Acute myeloid leukemia (AML) is a clonal disorder of myeloid hematopoiesis and a predominantly fatal hematopoietic malignancy with high heterogeneity [1]. Genetic heterogeneity has been appreciated since early karyotyping studies; somatic mutations have shown a comprehensive landscape of AML and contributed to disease classification and prognostic stratification [1-3]. However, a precise prognosis for nearly $50 \%$ of AML cases with a normal karyotype and patients with no mutations is still difficult to achieve [4]. DNA methylation, as the core and most widely studied epigenetic modification, is altered in numerous cancers and often correlates with clinically relevant information (i.e., subtypes, prognosis, and drug response) [5]. Indeed, aberrant DNA methylation is a common theme and a hallmark of AML [6]. Recent studies on genome-wide DNA methylation have emphasized the importance of dysregulated methylation profile in AML from biological and clinical views [7-11]. Aberrant DNA 
methylation has also been found suitable as a prognostic biomarker [7, 8, 10, 12-14]. However, the methylation techniques employed in these studies are difficult to apply in routine clinical practice [15].

Numerous DNA methylation detection techniques have thus far been developed, including those for genome scale and target enrichment methylation [15]. Currently, bisulfite treatment-based methylation microarray and next-generation sequencing (NGS) are commonly used for base resolution DNA methylomes, such as Illumina Human Methylation 450/850 BeadChip array (Illumina $450 \mathrm{~K} / 850 \mathrm{~K}$ array) [16-18], whole-genome bisulfite sequencing (WGBS) [19], reduced representation bisulfite sequencing (RRBS) [20], Agilent SureSelect Human Methyl-Seq (www.genomics.agilent.com) [21], and NimbleGen SeqCap Epi CpGiant (www.nimblegen.com) [22]. With high robustness and accuracy, DNA methylation analysis based on an NGS platform has been confirmed to be feasible and reliable in clinical diagnosis and precision medicine, particularly for highly heterogeneous diseases such as AML [23]. However, only $\sim 20 \%$ or less of cytosine-phosphate-guanine (CpGs) are variable across individuals or tissues [24]. WGBS is inefficient for large-scale population studies because it entails high costs and requires in-depth sequencing capacity to achieve sufficient coverage. Meanwhile, RRBS is limited to the restriction enzyme cutting site in a fixed region, which can potentially result in loss of data with the lowest genome coverage [15]. Agilent SureSelect allows only singlestrand capture of smaller target regions and requires larger amounts of input DNA, rendering it unsuitable for comprehensive genotype profiling [15]. Thus, alternative approaches to regulatory active functional methylome should be developed for comprehensive yet cost-effective identification of biologically and clinically relevant $\mathrm{CpGs}$ associated with complex diseases [25]. MethylC-capture sequencing (MCC-Seq) is an NGS capture approach that interrogates functional methylomes based on the NimbleGen SeqCap Epi CpGiant system with a unique design and long probes [26]. The technique provides comparable accuracy to alternative approaches but enables more efficient cataloguing of functional and diseaserelevant methylation variants for large-scale epigenomewide methylation studies $[25,26]$.

The current study presents MCC-Seq for the detection of prognostic methylation markers in AML and proposes a panel of 12 different functional DNA methylation genes.

\section{RESULTS}

\section{MCC-Seq and study control}

An outline of MCC-Seq is presented (Supplementary Figure 1). The sequence statistics obtained from 44 samples are summarized in Supplementary Table 1.
We targeted more than $5 \mathrm{Mb} \mathrm{CpG}$ sites of sequence covering genome-CpGs-scale with a total of 240,513 regions and a total size of $80 \mathrm{Mb}$. The average percentages of $\mathrm{CpG}$ sites with coverage depths of no less than $1 \times$, $5 \times, 10 \times$, and $20 \times$ were $92.73 \%, 80.32 \%, 67.20 \%$, and $43.81 \%$, respectively. All 44 DNA samples for MCC-Seq (in excess of $30 \times$ ) yielded $430 \mathrm{~Gb}$ of sequence data. The converted rate for all samples exceeded 99.5\%. A total of 58,147,036 (range: 40,663,694-79,302,058) clean reads on the average, were generated with an average of 95.03\% (range: $92.65 \%-98.05 \%$ ) clean Q30 base rate. Total mapping efficiency was $92.90 \%$ (range: $80.52 \%$ $96.40 \%$ ) and the average percentage of clean reads that mapped within the target CpGs was $72.32 \%$ (range: $38.54 \%-85.31 \%$ ).

We further performed a sample-based validation of MCC-Seq. Two single bone marrow samples were obtained from randomly selected patients with AML in relapse (C22 and $\mathrm{C} 23)$ and then prepared in replicate experiments (S22-Rep1 and S22-Rep2; S23-Rep1 and S23-Rep2). The effects of technical variability on methylation profiles were assessed by comparing the results of the replicates with independent captures and different degrees of multiplexing $(\geq 1 \times, 5 \times, 10 \times, 20 \times)$ (Supplementary Materials). The results indicated highly concordant methylation calls for overlapping $\mathrm{CpGs}$ between S22-Rep1 and S22-Rep2, and the correlation improved with increasing read depth cutoffs $(\mathrm{R}=0.959$, $0.971,0.978,0.985$ for the cutoffs of $1 \times, 5 \times, 10 \times, 20 \times)$, similar to S23-Rep1 and S23-Rep2 (Supplementary Figure 2).

In all subsequent population-based analyses in 37 samples with sequence depths $\geq 5 \times$, a total of 5,068,466 CpGs were yielded for further consideration with an average sequence depth of $23.6 \times$ and a minimum of $5 \times$ (Supplementary Figure 3). Approximately $43.9 \%$ of the captured $\mathrm{CpGs}$ showed a hypomethylated pattern ( $<20 \%$ methylation) and $48.5 \%$ exhibited hemi- to hypermethylated pattern ( $>50 \%$ methylation) in the 21 samples with de novo AML (Supplementary Figure 4). For subsequent DMR analysis, $\mathrm{a} \geq 10 \times$ coverage was required by removing those with below $10 \times$ coverage of sites over the 21 samples with de novo AML for distribution across all CpGs.

\section{Correlation between DNA methylation and clinical features}

We correlated some clinical features with the indicator of the DNA methylation level (DMI) (Supplementary Table 2) of genome-wide captured CpGs to determine whether a specific factor was associated with DMI in the 21 patients with de novo AML. Notably, the DMI was independent of the blast percentage of samples, age, gender, disease French-American-British (FAB) subtype, cytogenetic risk, molecular risk, and abundance 
of somatic mutations. Meanwhile, elder patients burdened a significantly higher DMI ( $\geq 50$ y vs. $<50$ y, $49.39 \%$ $\pm 3.43 \%$ vs. $46.75 \% \pm 2.31 \%, p=0.048$ ) (Figure 1). To further assess associations between DMI and blast percentage of samples, we prepared DNA samples derived from sorted and concentration graded bone marrow with blast percentages of $70 \%, 80 \%, 90 \%$ and $100 \%$ of patient C21 (Supplementary Materials). We found a high correlation between each percentage of blast samples $(\mathrm{R}$ $\geq 0.95$ ) with different read depth cutoffs (Supplementary Figure 5).

\section{Promoters have major different functional DNA methylation signatures}

As MCC-Seq has a genome-wide scale coverage of CpGs, the DNA methylation signature of different genomic features (i.e. all $\mathrm{CpGs}, \mathrm{CpG}$ islands, promoters,
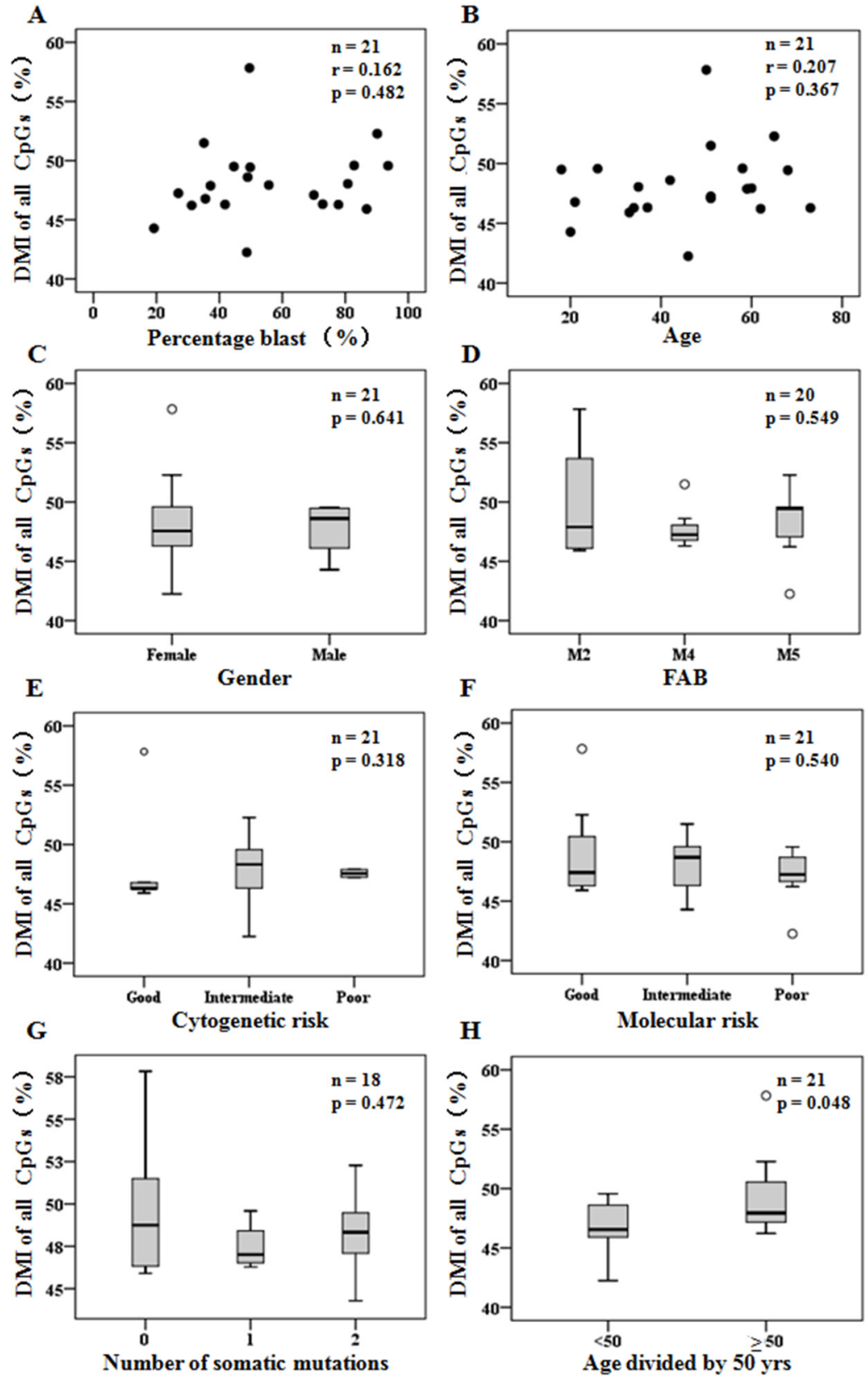

Figure 1: DMI of genome-wide captured CpGs detected in diagnosis were independent of clinical features. (A-B) Scatter plots of DMI (\%) compared to patient percentage blast of samples (A) and ages (B). (C-H) Box plots of DMI (\%) grouped by patient gender (C), FAB disease classification (D), cytogenetic risk status (E), molecular risk status (F), number of somatic mutations (G), and age divided by 50 years old $(\mathrm{H})$. Pearson correlation was used to determine r, and Student's $t$ test or one-way ANOVA was used for mean tests. 
exons, exon 1, introns, enhancers, 5 'untranslated region (5'UTR), etc.) (Supplementary Table 2) was compared among different clinical groups. Comparison of the DMI of these genomic features between de novo AML and normal bone marrow (NBM) indicated that only the DMI in promoters and enhancers were significantly higher in AML ( $p=0.025$ and $p=0.021$, respectively) (Figure 2A). Furthermore, a significant decrease in DMI in promoters $(p=0.018)$ was observed but not in enhancers $(p=0.145)$ by comparing the results from 8 paired samples (complete remission 1 (CR1) samples vs. diagnosis samples) (Figure 2B). Comparison of results from 3 other paired samples indicated that the DMI in promoters was similar between diagnosis and relapsed samples $(p=0.305)$. These results could indicate that the DNA methylation signature in promoters was representative in AML and associated with clinical response.

We compared the number of differentially methylated regions (DMRs) in mainly functional elements (promoter, exon 1, enhancer, 5'UTR) between de novo AML samples and NBM; the DMRs had the greatest number of promoters $(60.9 \%, 669 / 1099, p<0.001)$, which mainly consisted of hyper-DMRs $(75.0 \%, 502 / 669, p<$ 0.001) (Figure 2C). We also compared the DMRs among different cytogenetic risk groups and molecular risk groups within AMLs, then annotated genes that refered to these DMRs in functional elements (mainly in the promoter and enhancer regions) - that is, differentially methylated genes (DMGs) [22]. The results demonstrated that most DMGs were associated in promoters $(p<0.001)$ (Figure 2D, Supplementary Table 3). Thus, these results indicated that promoters have major different functional DNA methylation signatures, which were associated with established genetic prognostic stratifications. We subsequently explored prognostic methylation markers according to the DNA methylation signatures in promoters.

\section{Differentially methylated genes associated with established prognostic stratification}

A diagram illustrating the generation and validation of annotated DMGs according to the DMRs in promoter regions is summarized (Figure 3). To identify unfavorable genes with hyper DNA methylation and develop a molecular risk panel that incorporated both epigenetic and genetic prognostic information, we compared the DMRs in promoters among the different cytogenetic risk groups and molecular risk groups of 21 patients with de novo AML patients, both poor $v s$. intermediate, intermediate $v s$. good and poor vs. good subgroups. A total of 100 hyper-

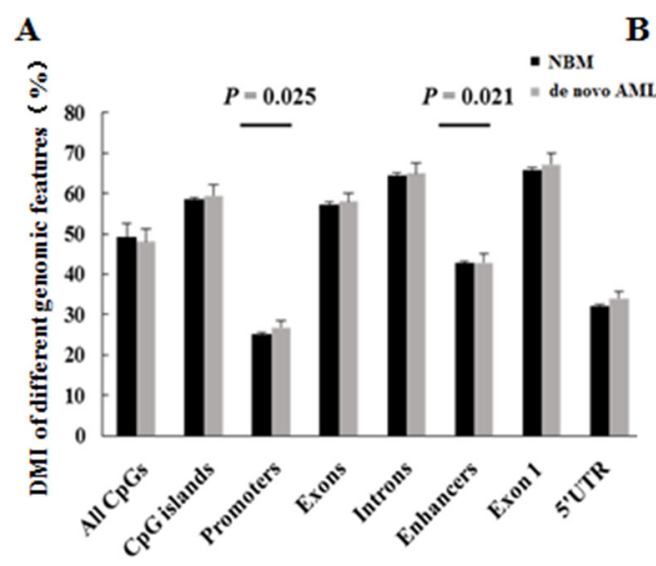

B

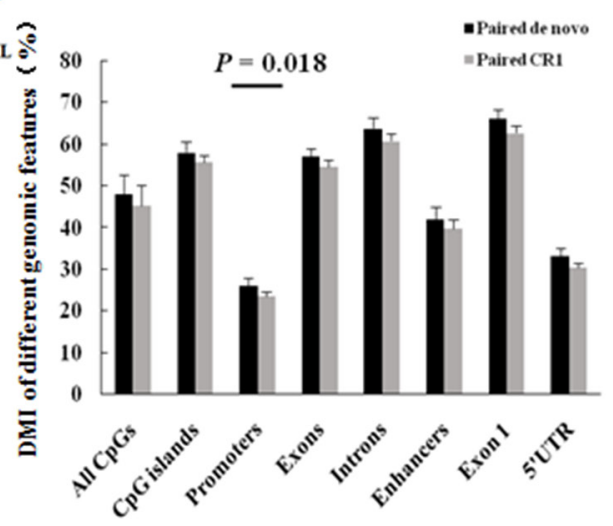

C

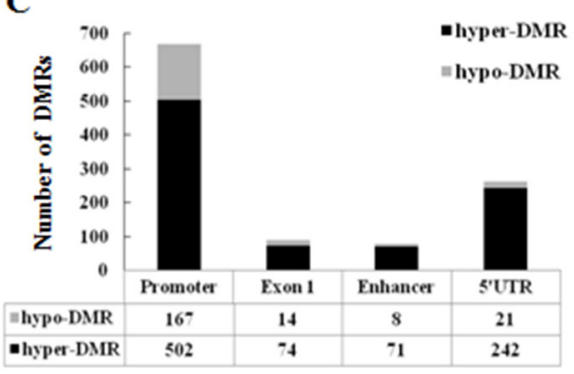

D

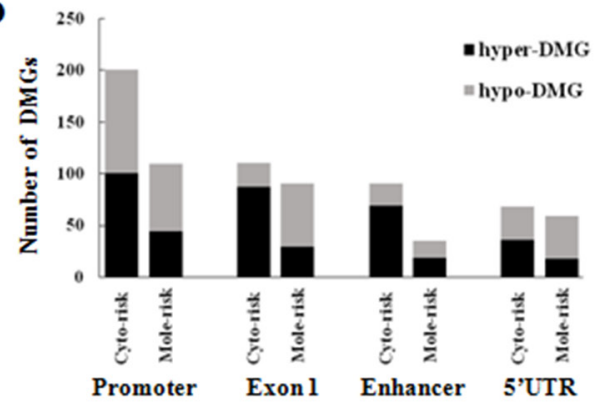

Figure 2: Promoters have major different functional DNA methylation signatures. (A) DMI of different genomic features compared between de novo AML and NBM samples. Student's $t$ test was used for mean tests. (B) DMI of different genomic features compared between 8 paired de novo AML and complete remission 1 (CR1) samples. (C) DMRs in functional elements between de novo AML and NBM samples. (D) DMGs in promoters compared among prognostic stratifications. Cyto-risk, Cytogenetics risk; Mole-risk, Molecular risk. 
DMGs were generated from the comparison of cytogenetic risks. Meanwhile, 44 hyper-DMGs were generated from the comparison of molecular risks (Supplementary Table 3). Subsequently, 18 hyper-DMGs were obtained by overlapping the 100 and 44 hyper-DMGs, both of which were associated with higher cytogenetic and molecular risks (Table 1).

Additional details, including the role of these 18 genes in cancer are summarized in Table 1. Except for 3 pseudogenes (GUCY1B2, HNRNPA1P33, and TUBA3FP), all of the remaining 15 genes showed the most involvement as tumor suppressor genes in distinct carcinomas, and four genes (TUBGCP2, PLEC, CLEC11A, and BARD1) were associated with AML. In addition, 7 genes (PLEC,
LSP1, IL18, IGF1, FOXD2, DEFB1, and CLEC11A) were reportedly regulated by DNA methylation. Gene Ontology (GO) and Kyoto Encyclopedia of Genes and Genomes (KEGG) pathways analysis were performed for the remaining 15 genes and 2 genes (MIR3150B and MIR4638) had no molecular function. We downloaded the DNA methylation datasets of AML for the 18 genes from The Cancer Genome Atlas (TCGA) data portal; however, no DNA methylation results on 5 genes (HNRNPA1P33, TUBA3F, MIR3150B, MIR4638, and PLEC) were available. Six genes were excluded due to pseudogenes, no molecular function, or no results in TCGA. A panel of 12 functional DMGs (BARD1, BCL9L, CLEC11A, DEFB1, FOXD2, IGF1, IL18, ITIH1, LSP1, P2RX6,

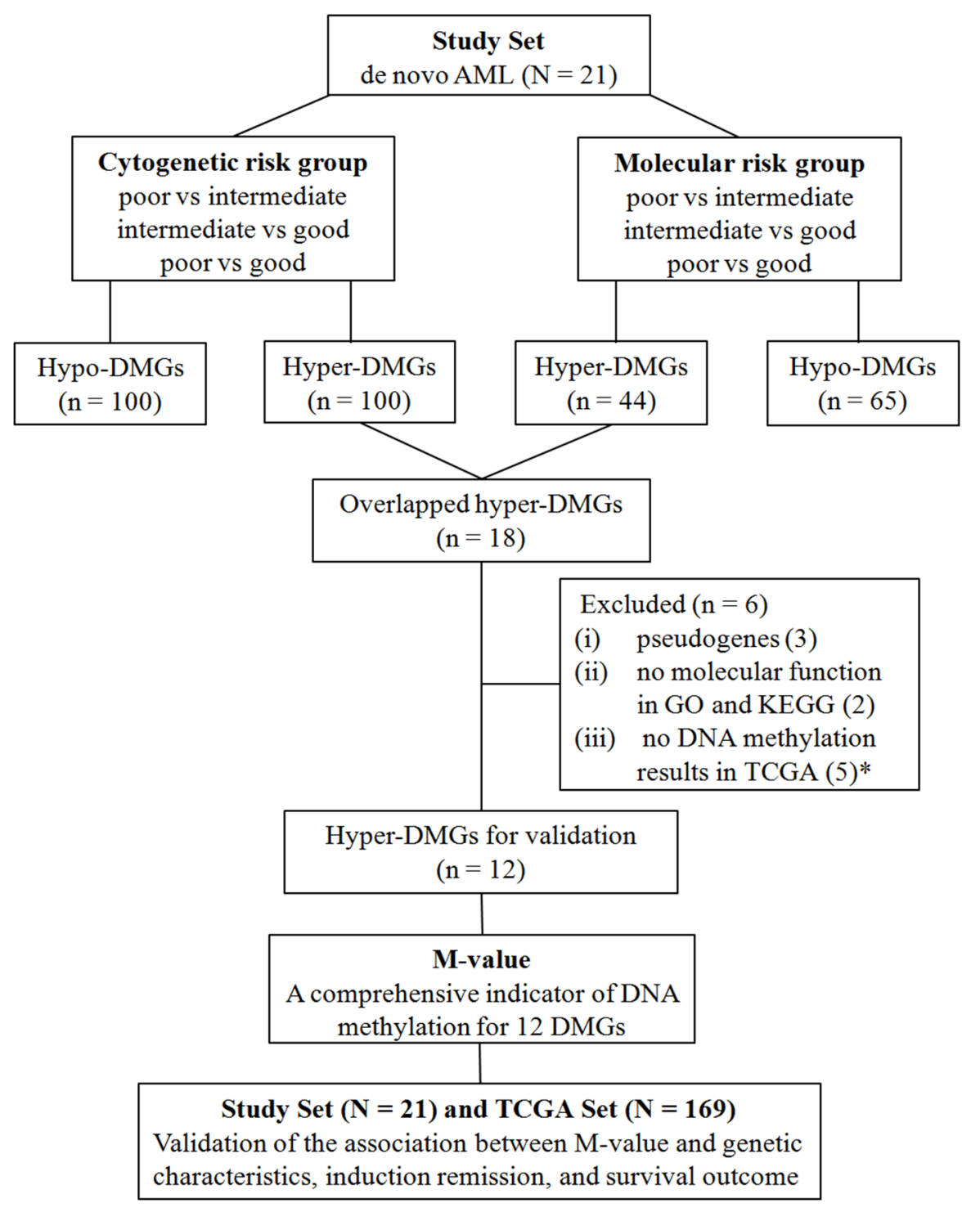

Figure 3: Diagram of the generation and validation of differentially methylated genes according to the DMRs in promoter. N, number of patients; n, number of genes; ${ }^{*}, 4$ genes were doubly counted, 2 of which were pseudogenes and the other 2 were without molecular functions in GO and KEGG analysis. 
Table 1: 18 hyper-DMGs associated with higher cytogenetic and molecular risks

\begin{tabular}{|c|c|c|c|c|}
\hline Gene symbol & Full name & Chr. location & Role in cancer & $\begin{array}{c}\text { ID in NCBI } \\
\text { gene database }\end{array}$ \\
\hline BARD1 & $\begin{array}{l}\text { BRCA1 associated } \\
\text { RING domain } 1\end{array}$ & $2 \mathrm{q} 35$ & $\begin{array}{l}\text { Down-regulation in MDS with progression } \\
\text { to AML, tumor suppressor genes [27] }\end{array}$ & 580 \\
\hline BCL9L & $\begin{array}{l}\text { B-cell CLL/lymphoma } \\
\text { 9-like }\end{array}$ & $11 \mathrm{q} 23.3$ & $\begin{array}{l}\text { Down-regulation associated with tumor cell } \\
\text { migration in ovarian cancer }[28]\end{array}$ & 283149 \\
\hline CLEC11A & $\begin{array}{l}\text { C-type lectin domain } \\
\text { family } 11 \text { member A }\end{array}$ & $19 q 13.33$ & $\begin{array}{l}\text { Hyper-methylation in pancreatic cancer } \\
\text { [29]; Associated with leukemia cell } \\
\text { proliferation [30] }\end{array}$ & 6320 \\
\hline DEFB1 & defensing beta 1 & $8 \mathrm{p} 23.1$ & $\begin{array}{l}\text { DNA methylation-mediated down- } \\
\text { regulation in prostate cancer [31]; tumor } \\
\text { suppressor genes [32] }\end{array}$ & 1672 \\
\hline FOXD2 & forkhead box D2 & $1 \mathrm{p} 33$ & $\begin{array}{l}\text { DNA methylation-mediated down- } \\
\text { regulation in colorectal cancer [33]; tumor } \\
\text { suppressor genes [34] }\end{array}$ & 2306 \\
\hline GUCY $1 B 2^{a}$ & $\begin{array}{l}\text { guanylate cyclase } 1 \\
\text { soluble subunit beta } 2 \\
\text { (pseudogene) }\end{array}$ & $13 q 14.3$ & Pseudogene & 2974 \\
\hline HNRNPA1P33 $3^{\mathrm{a}}$ & $\begin{array}{l}\text { heterogeneous nuclear } \\
\text { ribonucleoprotein A1 } \\
\text { pseudogene } 33\end{array}$ & $10 q 11.22$ & Pseudogene & 728643 \\
\hline IGF1 & $\begin{array}{l}\text { insulin like growth } \\
\text { factor } 1\end{array}$ & $12 \mathrm{q} 23.2$ & $\begin{array}{l}\text { Hyper-methylation involved in solid } \\
\text { tumor [35] }\end{array}$ & 3479 \\
\hline IL18 & interleukin 18 & $11 \mathrm{q} 23.1$ & Dual role involved in solid tumor $[36,37]$ & 360 \\
\hline ITIH1 & $\begin{array}{l}\text { inter-alpha-trypsin } \\
\text { inhibitor heavy chain } 1\end{array}$ & $3 \mathrm{p} 21.1$ & $\begin{array}{l}\text { Down-regulation involved in solid tumor } \\
\text { [38] }\end{array}$ & 3697 \\
\hline LSP1 & $\begin{array}{l}\text { lymphocyte-specific } \\
\text { protein } 1\end{array}$ & $11 \mathrm{p} 15.5$ & $\begin{array}{l}\text { Regulated by DNA methylation [39]; } \\
\text { Low expression in breast cancer [40] }\end{array}$ & 4046 \\
\hline MIR3150B & microRNA $3150 \mathrm{~b}$ & $8 \mathrm{q} 22.1$ & High expression in breast tumor [41] & 100500907 \\
\hline MIR4638 & microRNA 4638 & $5 q 35.3$ & High expression in breast tumor [41] & 100616342 \\
\hline P2RX6 & $\begin{array}{l}\text { purinergic receptor } \\
\mathrm{P} 2 \mathrm{X} 6\end{array}$ & $22 \mathrm{q} 11.21$ & $\begin{array}{l}\text { Regulated by p53, role in cancer } \\
\text { unknown [42] }\end{array}$ & 9127 \\
\hline $\mathrm{PLEC}^{\mathrm{a}}$ & plectin & $8 \mathrm{q} 24.3$ & $\begin{array}{l}\text { With genetic and epigenetic alterations in } \\
\text { AML [43] }\end{array}$ & 5339 \\
\hline RNASE3 & $\begin{array}{l}\text { ribonuclease A family } \\
\text { member } 3\end{array}$ & $14 \mathrm{q} 11.2$ & Low expression in pancreatic cancer [44] & 6037 \\
\hline TUBA3FPa & $\begin{array}{l}\text { tubulin alpha } 3 \mathrm{f} \\
\text { pseudogene }\end{array}$ & $22 \mathrm{q} 11.21$ & Pseudogene & 113691 \\
\hline TUBGCP2 & $\begin{array}{l}\text { tubulin gamma } \\
\text { complex associated } \\
\text { protein } 2\end{array}$ & $10 \mathrm{q} 26.3$ & High expression in AML [45] & 10844 \\
\hline
\end{tabular}

a These 6 genes were excluded for clinical validation. 
RNASE3, and TUBGCP2) associated with established genetic prognostic stratification were generated, and the prognostic significance was evaluated (Figure 3).

\section{Validation of 12-DMG panel for clinical implications}

We calculated the DNA methylation level of individual patients, based on the 12 DMGs. The M-value, which was the mean DMI of 12 DMGs for individual patients, was obtained. The clinical impact of M-value was then tested in 21 patients with AML in our study and validated in 169 patients with AML in the TCGA study.

\section{M-value is not associated with patient characteristics}

We correlated some clinical features with the M-value of the 12 DMGs to determine whether a specific factor was associated with the M-value both in the study set and TCGA set. Similarly, the M-value was independent of the age, gender, and blast percentage of the samples, similar to the DMI in all captured CpGs. However, patients with a higher cell differentiation subtype of FAB in the TCGA set burdened a higher M-value $(p<0.001)$ (Supplementary Figure 6).

\section{M-value is significantly associated with AML genetic characteristics}

Given the relationship of genetic characteristics and outcome in AML, we assessed the association of the M-value with AML genetic characteristics. In both the study set and the TCGA set, the M-value was shown to significantly improve with increasing cytogenetic and molecular risk stratifications (Table 2, Supplementary Figure 7). Moreover, unsupervised analysis using hierarchical clustering suggested that the patients in the study could be segregated into 3 groups (cluster $1, \mathrm{n}=$ 7; cluster 2, $\mathrm{n}=10$; cluster $3, \mathrm{n}=4$ ) according to the methylation profiles of these 12 DMGs (Figure 4A). The $\mathrm{M}$-values of these 3 clusters were significantly different $(26.59 \% \pm 4.36 \%$ vs. $41.08 \% \pm 4.72 \%$ vs. $68.47 \% \pm$ $4.33 \%, p=0.001)$. Notably, all 5 patients with goodrisk cytogenetics were in cluster 1 with a low M-value; 2 patients with poor-risk cytogenetic were in cluster 3 with a high M-value; and 10 of 14 patients with intermediaterisk cytogenetics were in cluster 2 with an intermediate $\mathrm{M}$-value. Four patients with cytogenetics intermediaterisk group (S01, S03, S15, S17-1) were assigned to low $\mathrm{M}$-value cluster 1 (S03, S17-1) and high M-value cluster 3 (S01, S15). Hierarchical clustering of 169 patients with AML from the TCGA data portal showed that patients could be segregated into 2 groups (cluster $1, \mathrm{n}=98$; cluster $2, \mathrm{n}=71$ ) according to the methylation profiles of these 12 DMGs (Figure 4B). The M-value of these 2 clusters were significantly different $(47.22 \% \pm 4.32 \%$ vs. $59.11 \% \pm 5.42 \%, p<0.001)$. Notably, 79 of 108 patients with intermediate-risk cytogenetic were assigned to cluster 1 with a low M-value, whereas 29 patients were assigned to cluster 2 with a high M-value, similarly to the case with molecular intermediate risk (79 to cluster 1 and 22 to cluster 2). These results suggested a high consistency between the DNA methylation profiles of 12 DMGs and the genetic signature for AML prognosis. Interestingly, some patients with intermediate-risk cytogenetics group could be distinguished by the M-value, which could further improve the prognosis stratification.

\section{M-value is associated with AML induction remission}

The mean M-value for patients in CR was lower than that for patients with no response (NR) $(37.42 \% \pm$ $15.79 \%$ vs. $49.69 \% \pm 12.51 \%, p=0.09$ ). To further assess the association between the M-value and CR, we divided the study set of 21 patients with AML to the low M-value group $(n=11)$ and the high M-value group $(n=10)$ according to the median of the M-value (Supplementary Table 4). Approximately, 90.9\% (10/11) of the patients achieved $\mathrm{CR}$ in the low M-value group, which was significantly higher than that in the high M-value group $40.0 \%, 4 / 10 ; p=0.024)$. Moreover, 5 of $6(83.3 \%)$ intermediate-risk AML (IR-AML) with a low M-value achieved CR, whereas only 3 of 8 (37.5\%) IR-AML with a high M-value achieved CR. We also found that the $\mathrm{CR}$ rate remained the same regardless of the regimen received by the patients - that is, the standard " $7+3$ " regimen or the "decitabine + cytarabine + aclarubicin + granulocyte colony-stimulating factor" (DCAG) regimen (Supplementary Table 4). These results indicated that patients with a low M-value were more likely to achieve $\mathrm{CR}$ in both total AML and IR-AML, independent of the induction regimen. This finding could not be verified in the TCGA set because of lack of information regarding induction remission response.

\section{M-value is associated with survival}

The relationship between M-value and survival was also examined both in the study set and the TCGA set (Figure 5). The patients were divided into the low M-value group and the high M-value group, as determined by the median of the M-value in each data set (Supplementary Table 5). First, the median overall survival (OS) / diseasefree survival (DFS) and 1-year cumulative OS/DFS of the 21 patients with AML were 23.8 months/not defined and $78.9 \% / 69.1 \%$, respectively (Table 3). A high M-value was associated with increased hazard for DFS alone (HR: 6.83, 95\%CI: 1.07-40.28) (Figure 5C). The values obtained for the low M-value group and the high M-value group were as follows: median OS, not defined and 14.93 months ( $p$ $=0.062)$; DFS, not defined and 10.97 months $(p=0.039)$; 1-year cumulative OS, $88.9 \%$ and $68.6 \%(p=0.145)$; and DFS, $90.9 \%$ and $30.0 \%(p<0.001)$, respectively (Figure $5 \mathrm{~A}, 5 \mathrm{C})$.

In the TCGA set, the high M-value group obtained a significantly poor OS than that of the low M-value group (median OS: 15.1 months vs. 16.4 months; HR: 1.491, 95\%CI: $1.043-2.151, p=0.038$ ) (Figure 5B). A trend 
Table 2: Correlation between M-value and genetic risk stratifications

\begin{tabular}{lcc}
\hline & Study set $(\mathbf{n}=\mathbf{2 1})$ & TCGA set $(\mathbf{n}=\mathbf{1 6 9})$ \\
\hline Cytogenetic risk & & \\
Good & $27.89 \% \pm 4.42 \%(\mathrm{n}=5)$ & $47.01 \% \pm 4.59 \%(\mathrm{n}=19)$ \\
Intermediate & $42.31 \% \pm 13.01 \%(\mathrm{n}=14)$ & $51.96 \% \pm 7.28 \%(\mathrm{n}=108)$ \\
Poor & $69.96 \% \pm 6.95 \%(\mathrm{n}=2)$ & $55.23 \% \pm 8.17 \%(\mathrm{n}=42)$ \\
$P$ value & 0.001 & 0.000 \\
Molecular risk & & $47.01 \% \pm 4.59 \%(\mathrm{n}=19)$ \\
Good & $32.01 \% \pm 6.69 \%(\mathrm{n}=8)$ & $52.19 \% \pm 7.41 \%(\mathrm{n}=101)$ \\
Intermediate & $45.61 \% \pm 18.76(\mathrm{n}=6)$ & $54.29 \% \pm 8.03 \%(\mathrm{n}=49)$ \\
Poor & $48.84 \% \pm 16.38(\mathrm{n}=7)$ & 0.002 \\
$P$ value & 0.047 & 59
\end{tabular}

in unfavorable prognosis for DFS without significant difference was observed in the high M-value group (median DFS: 13.8 months vs. 16.6 months; HR: 1.251, 95\%CI: 0.822-1.920, $p=0.296$ ) (Figure 5D). In addition, the high M-value group showed significantly lower 2-year OS and DFS (OS: 35.9\% vs. 45.9\%, $p=0.001$; DFS: $30.2 \%$ vs. $44.2 \%, p<0.001)$. The M-value based risk was meaningful for OS ( $p=0.038$, Figure 5B), and treatment with hematopoietic stem cell transplantation (HSCT) significantly improved OS (HSCT vs. no HSCT, median OS: 27 months vs. 7.7 months; HR: 0.4846, 95\%CI: 0.3214-0.6738, $p<0.0001$, Supplementary Figure $8 \mathrm{~A})$; thus, we assessed whether HSCT alters the M-value based risk. The results indicated that HSCT and survival outcomes were not adversely affected by the high M-value ( $p=0.271$, Supplementary Figure $8 \mathrm{~B}$ ), which suggested that the adverse prognostic effect of a high $\mathrm{M}$-value could be mitigated by HSCT.

\section{DISCUSSION}

In this study, genome-wide MCC-Seq was primarily used to detect prognostic DNA methylation markers in patients with AML. The sequencing profile showed a good converted rate (>99.5\%), clean Q30 base rate $(95.03 \%)$, mapping efficiency $(92.90 \%)$, and a high concordance of replicate experiments. These results indicated that MCCSeq is stable, reproducible and suitable for the analysis of bone marrow samples. By a series of comparative screening, a panel of 12 DMGs was identified with promoter hyper-DMRs associated with the outcome. A high M-value was associated with failure to achieve CR and poor survival, and its adverse prognostic effect could be mitigated by HSCT.

MCC-Seq is a NGS capture approach with the quantitative detection of DNA methylationn level (defined as DMI). To promote the clinical application of MCC-Seq in AML, we assessed the potential factors that could influence the DMI, which was found to be not associated with patient characteristics (e.g., age, gender, blast percentage of samples, FAB classification, etc.). Independent of blast percentage, DMI was further confirmed by bone marrow grading with different blast percentages from the same sample. These results were consistent with previous studies despite the differences in AML cohort, methods of detection and analysis, genomic regions analyzed, and DNA methylation index [10, 13, 46, 47]. Thus, BM blasts were regarded as a mere index of disease burden that, exerted no influence on methylome analysis. The reason was unclear and could suggest that DNA methylation assays reflect aberrant methylation in both blasts and more differentiated myeloid cells derived from leukemic precursors $[13,46]$. The independence and stability of DNA methylation analysis renders it suitable as a prognostic biomarker in AML.

DNA methylation is important for gene silencing via the hypermethylation of $\mathrm{CpG}$ islands in promoter regions [48]. The DNA promoter regions of critical tumor suppressor genes are inactivated via hypermethylation, which seems to significantly influence the pathogenesis and prognosis of AML $[49,50]$. Recent DNA methylome studies demonstrated that multi-locus DNA methylation assay in promoters can predict outcomes in de novo AML [7, 12-14]. Meanwhile, some studies revealed more diverse DNA methylation functions dependent on genomic location, particularly in enhancers $[10,24,48,51]$. In the current study, with a genome-wide scale coverage of $\mathrm{CpGs}$ by MCC-Seq, the DNA methylation signature of different genomic features was evaluated to avoid regional bias. Consequently, promoters obtained a significantly higher DMI in de novo AML $(p=0.025)$ and was significantly lower in CR1 $(p=0.018)$. The most number of DMRs and DMGs were distributed in promoters compared with other regions $(p<0.001)$. These results collectively 

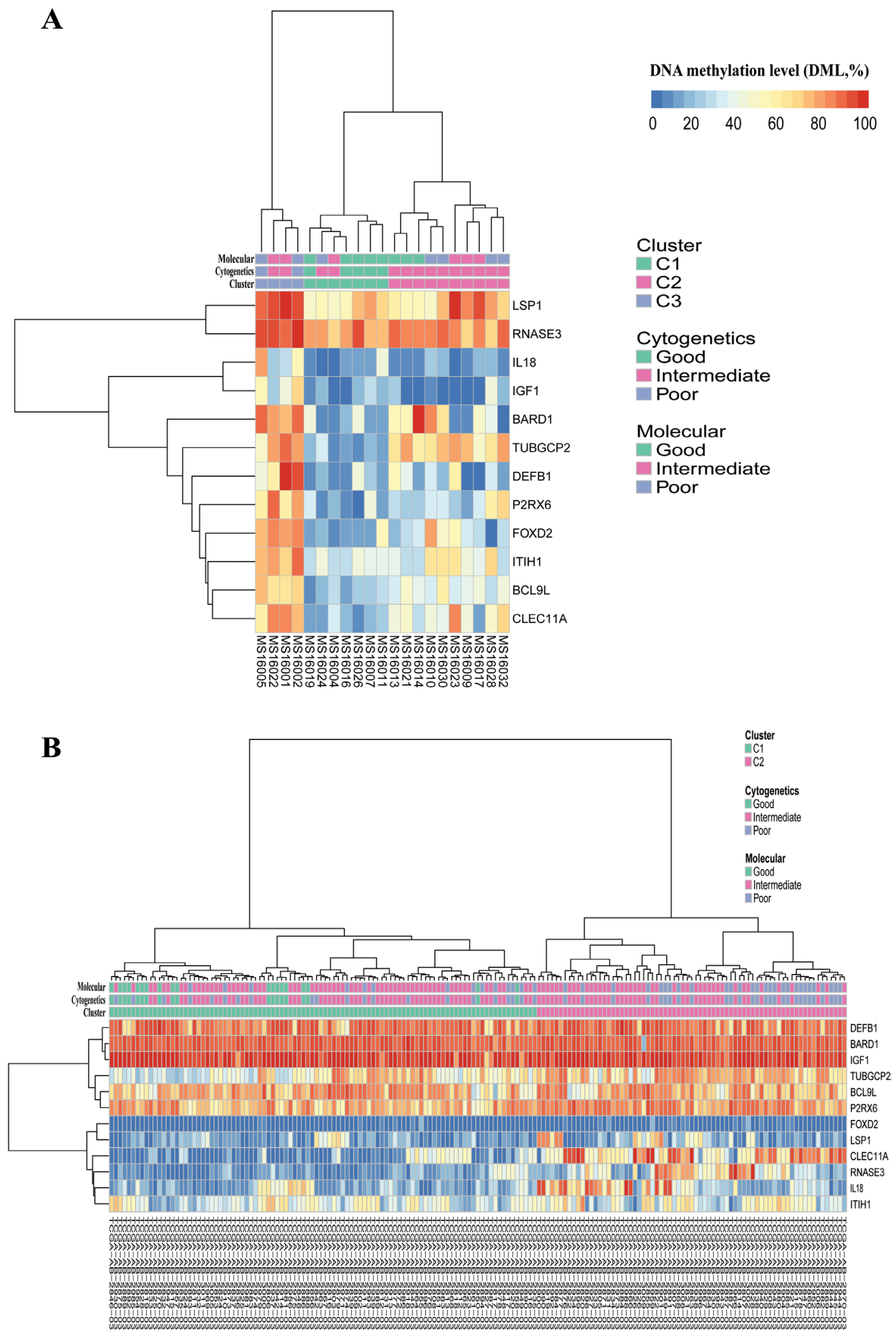

Figure 4: Hierarchical clustering of the study set $(\mathbf{A}, n=21)$ and the TCGA set $(\mathbf{B}, n=169)$ according to their methylation profiles of the 12 DMGs grouped by clusters, cytogenetics risk stratifications and molecular risk stratifications, respectively. 
indicated that promoters have major different functional DNA methylation signatures in AML, as demonstrated in previous and recent studies [6, 9, 10, 12, 47].

AML is a complex disease with genetic and epigenetic changes $[3,10]$. However, classification and prognostication of the disease for AML patients have thus far been largely dependent on cytogenetic and genetic testing (recurrent somatic mutations), whereas epigenetic changes, including DNA methylation, have not been considered [1]. Marcucci et al. were the first to integrate genetic and epigenetic information for prognostication and treatment response prediction in AML [12]. They reported a gene expression score involving 7 oncogenes associated with somatic mutations and DNA methylation for a meaningful prognosis. The limitation was that the genes were derived from a cohort of older patients with cytogenetically normal AML (CN-AML) and only validated in CN-AML sets. The present study encompassed both genetic (cytogenetic risk and molecular risk) and epigenetic (DNA methylation) information from a new standpoint to develop a novel prognostic gene panel in all non AML-M3 subtypes. On the basis of cytogenetic and molecular risk stratification, we identified DMRs and DMGs to select hypermethylated genes in higherrisk stratification subgroups. To ensure the accuracy and reliability of these hyper-DMGs, 18 overlapping hyperDMGs associated with both increased risk of cytogenetics and molecular stratification were obtained. A panel of 12 DMGs with a strict selection process was formed. Most of these genes acted as tumor suppressors in cancer and were regulated by DNA methylation (Table 1), which was consistent with our screening process (genes hypermethylated in the higher-risk group).

The prognostic value of these 12 DMGs was evaluated in the study set and the TCGA set. The M-value of the 12 DMGs was significantly associated with AML genetic characteristics. Consistent with previous studies, the M-value obtained in the present study not only predicted CR rates and DFS or OS duration in all patients with AML but also represented the prognostic value for patients in CR with IR-AML [10, 12-14]. Luskin et al. did not assess whether HSCT alters their DNA methylationbased risk because of insufficient power in their cohort [13]. In the current study, we investigated this issue by using the M-value-based risk in 81 patients with HSCT from the TCGA set. The results indicated that HSCT may mitigate the adverse prognostic effect of high M-value. High M-values were associated with failure to achieve CR and with poor OS or DFS; however, patients with high M-values could benefit from HSCT, which is clearly an essential area of future investigation.

In summary, genome-CpGs-scale detection of prognostic methylation markers by MCC-Seq is feasible and revealed an M-value for 12 genes that could be used as a valuable biomarker for risk stratification. The M-value is suitable for all patients with AML, particularly those without genetic and molecular markers. It is expected to be used as a biomarker to guide demethylation therapy. Patients predicted to have poor outcomes based on high M-value may benefit from more intensive post-remission treatment (e.g., HSCT) or enrollment in a clinical trial. None of these 12 genes were reported in other prognostic
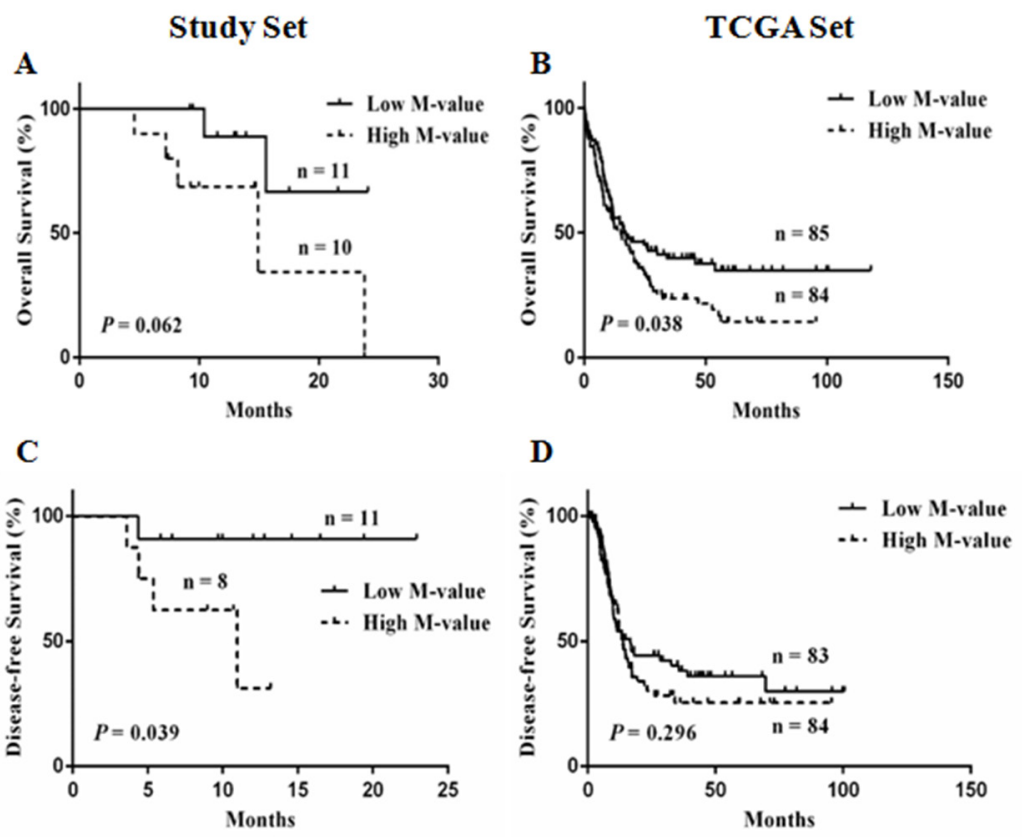

Figure 5: Kaplan-Meier curves for low and high M-value groups. (A, C) overall survival (OS) and disease-free survival (DFS) of the study set; (B, D) OS and DFS of the TCGA set. 
Table 3: Patient and sample characteristics

Patient characteristics

Age (y)

Male sex: no. (\%)

AML FAB subtype: no. (\%)

AML with maturation: M2

Acute myelomonocytic leukemia: M4

Acute monoblastic or monocytic leukemia: M5

Acute erythroid leukemia: M6

AML WHO subtype: no. (\%)

AML, NOS

AML with $\mathrm{t}(8 ; 21)(\mathrm{q} 22 ; \mathrm{q} 22.1)$

AML with MDS-related changes

AML with biallelic mutations of CEBPA

AML with CBFB-MYH11

AML with mutated NPM1

Bone marrow blasts at diagnosis:\%

Normal cytogenetic profile: no. (\%)

Whitecell count at diagnosis: per $\mathrm{mm}^{3}$

Mean

Median (range)

Cytogenetic risk group: no. (\%)

Good

Intermediate

Poor

Molecular risk group: no. (\%)

Good

Intermediate

Poor

Remission induction: no. (\%)

$7+3^{*}$

$\mathrm{DCAG}^{\#}$

Response: no. (\%)

Complete remission (CR)

No response (NR)

Median follow-up (OS/DFS)

1-year Cumulative OS

1-year Cumulative DFS

Median OS

Median DFS
$\mathbf{N}=\mathbf{2 1}$

$45.71 \pm 16.50$

7 (33.3)

4 (19.0)

9 (42.9)

$7(33.3)$

$1(4.8)$

8 (38.1)

3 (14.3)

$2(9.5)$

$4(19.1)$

$2(9.5)$

$2(9.5)$

$64.46 \pm 19.98$

9 (42.9)

$21,822 \pm 34,371$

$11,390(590,137,630)$

5 (23.8)

$14(66.7)$

$2(9.5)$

8 (38.1)

6 (28.6)

$7(33.3)$

9 (42.9)

$12(57.1)$

14 (66.7)

7 (33.3)

12.9 Months/ 10.0 Months

$78.9 \% \pm 9.6 \%$

$69.1 \pm 12.3 \%$

23.8 Months 


\begin{tabular}{lc}
\hline Sample characteristics & $\mathbf{N}=\mathbf{4 4}$ \\
\hline NBM & $\mathrm{N}=\mathbf{5}$ \\
AML samples & $\mathrm{N}=39$ \\
De novo & 21 \\
Paired complete remission (cycle 1) & 8 \\
Paired relapsed & 3 \\
Concentration gradients & 3 \\
Reduplicate & 4 \\
\hline
\end{tabular}

* " $7+3$ ", standard induction regimens based on a backbone of cytarabine plus an anthracycline, with details according to the NCCN guideline for AML.

\# DCAG, decitabine $20 \mathrm{mg} / \mathrm{m}^{2} \mathrm{~d} 1-5$, cytarabine $10 \mathrm{mg} / \mathrm{m}^{2} \mathrm{q} 12 \mathrm{~h} \mathrm{~d} 1-5$, aclarubicin $20 \mathrm{mg} \mathrm{d} 1,3,5$, G-CSF $300 \mu \mathrm{g} / \mathrm{d}$ until recovery from neutropenia.

FAB, French-American-British; WHO, World Health Organization; OS, overall survival; DFS, disease-free survival;

NBM, normal bone marrow.

studies based on methylation status $[7,9,10,13,14]$. However, methodologic differences prevented a direct, meaningful comparison of our integrated genetic risk and different DNA methylation prognostic markers with previously reported results. Our conclusions are currently limited, given the small size of the cohort study. The prognostic value of the M-value has to be verified in future larger-scale studies.

\section{MATERIALS AND METHODS}

\section{Ethics statement}

The study protocol was approved by the author's institutional ethics committee, the Ethics Committee of the General Hospital of Chinese People's Liberation Army, and was conducted in accordance with the Declaration of Helsinki. Written informed consent was obtained from each participant prior to specimen collection.

\section{Patients and samples}

A total of 21 patients with de novo AML, 2 patients with AML in relapse, and 5 healthy donors for related allogeneic HSCT who visited our hematology department between August 2014 and June 2016 were enrolled in the study. DNA samples from bone marrow and clinical information were collected (Supplementary Materials). A total of 35 samples from the bone marrow of 21 adult patients with AML (non AML-M3) were obtained. In addition, four replicate samples were obtained from another set of 2 randomly selected relapsed AML patients, and 5 samples from NBM were obtained. The characteristics of the patients with de novo AML and all samples are fully described in Table 3 and Supplementary Table 6. The diagnosis and prognosis of AML were based on World Health Organization 2016 classification and the AML guidelines of the National Comprehensive Cancer Network (NCCN; AML, Version 1.2017; http://www. nccn.org/). The 21 patients consisted of 14 women and 7 men with a median age of $50 \mathrm{y}$ (range, 18-73 y). Median follow-up was 12.9 months (range, 4.6-24.1 months) for OS and 10.0 months (range, 3.6-22.9 months) for DFS.

\section{MCC-Seq protocol}

A total of 44 DNA samples were used for MCCSeq (Table 3). The concentration and integrity of DNA were detected by electrophoresis to confirm the quality. In MCC-Seq, a whole-genome methylation sequencing library is prepared with the qualified DNA samples, bisulfate-converted, and amplified, followed by a capture enriched for targeted bisulfite-converted DNA fragments. This process is achieved using the novel SeqCap Epi probe design platform developed by Roche NimbleGen. This platform enables the capture of double-stranded targets regardless of their methylated state via high-density tiling of probes $[22,25]$. Each capture was sequenced on a single lane of the 125 bp paired-end Illumina HiSeq2500 System (Supplementary Materials).

\section{MCC-Seq methylation analysis}

The glossaries used in this study are summarized in Supplementary Table 2. Raw sequence reads were filtered to remove adapter contamination and poor-quality reads. Clean sequences were first mapped to the human genome (build GRCh37) by using Bismark (v0.10.1; parameters: -pe, -bowtie2, -directional, -unmapped). Methylation calls were extracted after duplicate sequences had been excluded. DMRs were analyzed in R 3.1 .0 by using the methylKit package. The minimum read coverage 
to call a methylation status for a base was set to 5. All off-target reads were removed. The methylation level at each site was determined by dividing the number of reads supporting methylation for that site by the total number of reads covering that site. $\mathrm{CpGs}$ were included in subsequent analyses if the number of sequence reads was 5 or greater. Data visualization and analysis were performed using Integrative Genomics Viewer, custom R, and Perl scripts (Supplementary Materials).

To ensure the reliability of the sequencing results without bias, both the technicians and bioinformatics analyst were blinded to the clinical information of the samples.

\section{Analyses of DNA methylation data from TCGA AML cohort}

TCGA performed profiling using Illumina Infinium HumanMethylation450 BeadChip for 194 samples of 200 clinically annotated adult cases of de novo AML [3]. Clinical data and DNA methylation datasets for the AML cohort are publicly available through the TCGA data portal (https://tcga-data.nci.nih.gov/tcga/). The MCC-Seq platform targets the same set of genes as the $450 \mathrm{~K}(99 \%$ of RefSeq genes) [15, 22]; thus, we included 169 patients with non AML-M3 with complete cytogenetic/molecular risk information and DNA methylation profiles into this study to validate the correlation of DNA methylation and clinical features (Supplementary Table 7).

\section{Statistical analyses}

The demographics and characteristics were summarized using descriptive statistics. Student's $t$ test or the Mann-Whitney U-test were used to compare continuous variables. Categorical variables were compared using the Fisher's exact test or Chi-square test. Outcome measures were assessed using Kaplan-Meier estimates in a univariate analysis. OS was defined as the time from diagnosis to death from any cause or last follow-up. DFS was defined as the time from CR to the date of relapse, death, or last follow-up. A two-sided p-value $<0.05$ was considered statistically significant. All statistical analyses were performed with SPSS software version 19.0 (IBM Corp., Armonk, NY, USA), GraphPad Prism 6 (GraphPad Software Inc., San Diego, California, USA).

\section{Author contributions}

YL and LY designed the research. YL, HMZ, QYX, XWW, and LY performed the research. YL and HMZ performed the statistical analysis and biostatistics. YL, HMZ, and QYX drafted the manuscript. JG and CJC analyzed or interpreted the data. NL, YHL, and LY critically revised the manuscript. YJ, LLW, LZ, JL and GFC provided valuable advice and also revised the manuscript. All authors read and approved the final manuscript.

\section{ACKNOWLEDGMENTS}

The authors are grateful to Lili Cai from Annoroad Gene Technology Co. Ltd for the preparation of the graphical illustrations.

\section{CONFLICTS OF INTEREST}

The authors declare there is no conflicts of interests.

\section{FUNDING}

This work was supported by the National Natural Science Foundation of China (Grant nos. 8167016, $81370635,81170518,81270611,81570137,81470010$ and 81400135), Capital Medical Development Scientific Research Fund (SF2001-5001-07), Beijing Natural Science Foundation (7151009), National Public Health Grant Research Foundation (No.201202017) and The Capital of the Public Health Project (Z111107067311070). The funders had no role in the study design, data collection and analysis, decision to publish, or preparation of the manuscript.

\section{REFERENCES}

1. Papaemmanuil E, Gerstung M, Bullinger L, Gaidzik VI, Paschka P, Roberts ND, Potter NE, Heuser M, Thol F, Bolli N, Gundem G, Van Loo P, Martincorena I, et al. Genomic classification and prognosis in acute myeloid leukemia. N Engl J Med. 2016; 374: 2209-21. https://doi.org/10.1056/ NEJMoa1516192.

2. Testa JR, Mintz U, Rowley JD, Vardiman JW, Golomb HM. Evolution of karyotypes in acute nonlymphocytic leukemia. Cancer Res. 1979; 39: 3619-27.

3. Cancer Genome Atlas Research Network. Genomic and epigenomic landscapes of adult de novo acute myeloid leukemia. N Engl J Med. 2013; 368: 2059-74. https://doi. org/10.1056/NEJMoa1301689.

4. Bullinger L, Kronke J, Schon C, Radtke I, Urlbauer K, Botzenhardt U, Gaidzik V, Cario A, Senger C, Schlenk RF, Downing JR, Holzmann K, Dohner K, et al. Identification of acquired copy number alterations and uniparental disomies in cytogenetically normal acute myeloid leukemia using high-resolution single-nucleotide polymorphism analysis. Leukemia. 2010; 24: 438-49. https://doi.org/10.1038/ leu.2009.263.

5. Baylin SB, Jones PA. A decade of exploring the cancer epigenome - biological and translational implications. Nat Rev Cancer. 2011; 11: 726-34. https://doi.org/10.1038/ $\operatorname{nrc3} 130$. 
6. Gutierrez SE, Romero-Oliva FA. Epigenetic changes: a common theme in acute myelogenous leukemogenesis. J Hematol Oncol. 2013; 6: 57. https://doi. org/10.1186/1756-8722-6-57.

7. Figueroa ME, Lugthart S, Li Y, Erpelinck-Verschueren C, Deng X, Christos PJ, Schifano E, Booth J, van Putten W, Skrabanek L, Campagne F, Mazumdar M, Greally JM, et al. DNA methylation signatures identify biologically distinct subtypes in acute myeloid leukemia. Cancer Cell. 2010; 17: 13-27. https://doi.org/10.1016/j.ccr.2009.11.020.

8. Deneberg S, Grovdal M, Karimi M, Jansson M, Nahi H, Corbacioglu A, Gaidzik V, Dohner K, Paul C, Ekstrom TJ, Hellstrom-Lindberg E, Lehmann S. Gene-specific and global methylation patterns predict outcome in patients with acute myeloid leukemia. Leukemia. 2010; 24: 932-41. https://doi.org/10.1038/leu.2010.41.

9. Deneberg S, Guardiola P, Lennartsson A, Qu Y, Gaidzik V, Blanchet O, Karimi M, Bengtzen S, Nahi H, Uggla B, Tidefelt U, Hoglund M, Paul C, et al. Prognostic DNA methylation patterns in cytogenetically normal acute myeloid leukemia are predefined by stem cell chromatin marks. Blood. 2011; 118: 5573-82. https://doi.org/10.1182/ blood-2011-01-332353.

10. Li S, Garrett-Bakelman FE, Chung SS, Sanders MA, Hricik T, Rapaport F, Patel J, Dillon R, Vijay P, Brown AL, Perl AE, Cannon J, Bullinger L, et al. Distinct evolution and dynamics of epigenetic and genetic heterogeneity in acute myeloid leukemia. Nat Med. 2016; 22: 792-9. https://doi. org/10.1038/nm.4125.

11. Li Y, Xu Q, Lv N, Wang L, Zhao H, Wang X, Guo J, Chen C, Li Y, Yu L. Clinical implications of genome-wide DNA methylation studies in acute myeloid leukemia. J Hematol Oncol. 2017; 10: 41. https://doi.org/10.1186/ s13045-017-0409-z.

12. Marcucci G, Yan P, Maharry K, Frankhouser D, Nicolet D, Metzeler KH, Kohlschmidt J, Mrozek K, Wu YZ, Bucci D, Curfman JP, Whitman SP, Eisfeld AK, et al. Epigenetics meets genetics in acute myeloid leukemia: clinical impact of a novel seven-gene score. J Clin Oncol. 2014; 32: 54856. https://doi.org/10.1200/JCO.2013.50.6337.

13. Luskin MR, Gimotty PA, Smith C, Loren AW, Figueroa ME, Harrison J, Sun Z, Tallman MS, Paietta EM, Litzow MR, Melnick AM, Levine RL, Fernandez HF, et al. A clinical measure of DNA methylation predicts outcome in de novo acute myeloid leukemia. JCI Insight. 2016. https:// doi.org/10.1172/jci.insight.87323.

14. Dinardo CD, Luskin MR, Carroll M, Smith C, Harrison J, Pierce S, Kornblau S, Konopleva M, Kadia T, Kantarjian H, Wertheim GB, Master SR. Validation of a clinical assay of multi-locus DNA methylation for prognosis of newly diagnosed AML. Am J Hematol. 2017; 92: E14-E5. https:// doi.org/10.1002/ajh.24597.

15. Sun Z, Cunningham J, Slager S, Kocher JP. Base resolution methylome profiling: considerations in platform selection, data preprocessing and analysis. Epigenomics. 2015; 7: 813-28. https://doi.org/10.2217/epi.15.21.

16. Dedeurwaerder S, Defrance M, Calonne E, Denis H, Sotiriou C, Fuks F. Evaluation of the Infinium Methylation 450K technology. Epigenomics. 2011; 3: 771-84. https:// doi.org/10.2217/epi.11.105.

17. Sandoval J, Heyn H, Moran S, Serra-Musach J, Pujana MA, Bibikova M, Esteller M. Validation of a DNA methylation microarray for 450,000 $\mathrm{CpG}$ sites in the human genome. Epigenetics. 2011; 6: 692-702. http://dx.doi.org/10.4161/ epi.6.6.16196.

18. Moran S, Arribas C, Esteller M. Validation of a DNA methylation microarray for $850,000 \mathrm{CpG}$ sites of the human genome enriched in enhancer sequences. Epigenomics. 2016; 8: 389-99. https://doi.org/10.2217/epi.15.114.

19. Bock C, Tomazou EM, Brinkman AB, Muller F, Simmer F, Gu H, Jager N, Gnirke A, Stunnenberg HG, Meissner A. Quantitative comparison of genome-wide DNA methylation mapping technologies. Nat Biotechnol. 2010; 28: 1106-14. https://doi.org/10.1038/nbt.1681.

20. Wang L, Sun J, Wu H, Liu S, Wang J, Wu B, Huang S, Li N, Wang J, Zhang X. Systematic assessment of reduced representation bisulfite sequencing to human blood samples: a promising method for large-sample-scale epigenomic studies. J Biotechnol. 2012; 157: 1-6. https:// doi.org/10.1016/j.jbiotec.2011.06.034.

21. Agilent Technologies: SureSelect Methyl-Seq. www. genomics.agilent.com.

22. NimbleGen. www.nimblegen.com.

23. Blueprint Consortium. Quantitative comparison of DNA methylation assays for biomarker development and clinical applications. Nat Biotechnol. 2016; 34: 726-37. https://doi. org/10.1038/nbt.3605.

24. Ziller MJ, Gu H, Muller F, Donaghey J, Tsai LT, Kohlbacher O, De Jager PL, Rosen ED, Bennett DA, Bernstein BE, Gnirke A, Meissner A. Charting a dynamic DNA methylation landscape of the human genome. Nature. 2013; 500: 477-81. https://doi.org/10.1038/nature12433.

25. Allum F, Shao X, Guenard F, Simon MM, Busche S, Caron M, Lambourne J, Lessard J, Tandre K, Hedman AK, Kwan T, Ge B; Multiple Tissue Human Expression Resource Consortium, et al. Characterization of functional methylomes by next-generation capture sequencing identifies novel disease-associated variants. Nat Commun. 2015; 6: 7211. https://doi.org/10.1038/ncomms8211.

26. Walker DL, Bhagwate AV, Baheti S, Smalley RL, Hilker CA, Sun Z, Cunningham JM. DNA methylation profiling: comparison of genome-wide sequencing methods and the Infinium Human Methylation 450 Bead Chip. Epigenomics. 2015; 7: 1287-302. https://doi.org/10.2217/EPI.15.64.

27. Mahadevan D, DiMento J, Croce KD, Riley C, George B, Fuchs D, Mathews T, Wilson C, Lobell M. Transcriptosome and serum cytokine profiling of an atypical case of myelodysplastic syndrome with progression to acute 
myelogenous leukemia. Am J Hematol. 2006; 81: 779-86. https://doi.org/10.1002/ajh.20690.

28. Li B, Cai S, Zhao Y, He Q, Yu X, Cheng L, Zhang Y, Hu $\mathrm{X}, \mathrm{Ke} \mathrm{M}$, Chen S, Zou M. Nerve growth factor modulates the tumor cells migration in ovarian cancer through the WNT/beta-catenin pathway. Oncotarget. 2016; 7: 8102648. https://doi.org/10.18632/oncotarget.13186.

29. Kisiel JB, Raimondo M, Taylor WR, Yab TC, Mahoney DW, Sun Z, Middha S, Baheti S, Zou H, Smyrk TC, Boardman LA, Petersen GM, Ahlquist DA. New DNA methylation markers for pancreatic cancer: discovery, tissue validation, and pilot testing in pancreatic juice. Clin Cancer Res. 2015; 21: 4473-81. https://doi.org/10.1158/1078-0432. CCR-14-2469.

30. Hiraoka A. Leukemia cell lines require self-secreted stem cell growth factor (SCGF) for their proliferation. Leuk Res. 2008; 32: 1623-5. https://doi.org/10.1016/j. leukres.2008.01.003.

31. Lee J, Han JH, Jang A, Kim JW, Hong SA, Myung SC. DNA methylation-mediated downregulation of DEFB1 in prostate cancer cells. PLoS One. 2016; 11: e0166664. https://doi.org/10.1371/journal.pone.0166664.

32. Bullard RS, Gibson W, Bose SK, Belgrave JK, Eaddy AC, Wright CJ, Hazen-Martin DJ, Lage JM, Keane TE, Ganz TA, Donald CD. Functional analysis of the host defense peptide Human Beta Defensin-1: new insight into its potential role in cancer. Mol Immunol. 2008; 45: 839-48. https://doi.org/10.1016/j.molimm.2006.11.026.

33. Conesa-Zamora P, Garcia-Solano J, Turpin Mdel C, Sebastian-Leon P, Torres-Moreno D, Estrada E, Tuomisto A, Wilce J, Makinen MJ, Perez-Guillermo M, Conesa A. Methylome profiling reveals functions and genes which are differentially methylated in serrated compared to conventional colorectal carcinoma. Clin Epigenetics. 2015; 7: 101. https://doi.org/10.1186/s13148-015-0128-7.

34. Sulman EP, White PS, Brodeur GM. Genomic annotation of the meningioma tumor suppressor locus on chromosome 1p34. Oncogene. 2004; 23: 1014-20. https://doi. org/10.1038/sj.onc. 1206623 .

35. Zhang C, Zhao H, Li J, Liu H, Wang F, Wei Y, Su J, Zhang D, Liu T, Zhang Y. The identification of specific methylation patterns across different cancers. PLoS One. 2015; 10: e0120361. https://doi.org/10.1371/journal. pone. 0120361 .

36. Jurecekova J, Babusikova E, Kmetova Sivonova M, Drobkova H, Petras M, Kliment J, Halasova E. Association between interleukin-18 variants and prostate cancer in Slovak population. Neoplasma. 2017; 64: 148-55. https:// doi.org/10.4149/neo_2017_119.

37. Guo X, Zheng L, Jiang J, Zhao Y, Wang X, Shen M, Zhu F, Tian R, Shi C, Xu M, Li X, Peng F, Zhang H, et al. Blocking NF-kappaB is essential for the immunotherapeutic effect of recombinant IL18 in pancreatic cancer. Clin Cancer Res. 2016; 22: 5939-50. https://doi.org/10.1158/1078-0432. CCR-15-1144.
38. Hamm A, Veeck J, Bektas N, Wild PJ, Hartmann A, Heindrichs U, Kristiansen G, Werbowetski-Ogilvie T, Del Maestro R, Knuechel R, Dahl E. Frequent expression loss of Inter-alpha-trypsin inhibitor heavy chain (ITIH) genes in multiple human solid tumors: a systematic expression analysis. BMC Cancer. 2008; 8: 25. https://doi. org/10.1186/1471-2407-8-25.

39. Chandra S, Baribault C, Lacey M, Ehrlich M. Myogenic differential methylation: diverse associations with chromatin structure. Biology (Basel). 2014; 3: 426-51. https://doi.org/10.3390/biology3020426.

40. Poorhosseini SM, Hashemi M, Alipour Olyaei N, Izadi A, Moslemi E, Ravesh Z, Hashemi-Gorji F, Kheiri HR, Yassaee VR. New gene profiling in determination of breast cancer recurrence and prognosis in Iranian women. Asian Pac J Cancer Prev. 2016; 17: 155-60. http://dx.doi. org/10.7314/APJCP.2016.17.S3.155.

41. Persson H, Kvist A, Rego N, Staaf J, Vallon-Christersson J, Luts L, Loman N, Jonsson G, Naya H, Hoglund M, Borg A, Rovira C. Identification of new microRNAs in paired normal and tumor breast tissue suggests a dual role for the ERBB2/Her2 gene. Cancer Res. 2011; 71: 78-86. https:// doi.org/10.1158/0008-5472.CAN-10-1869.

42. Urano T, Nishimori H, Han H, Furuhata T, Kimura Y, Nakamura Y, Tokino T. Cloning of P2XM, a novel human P2X receptor gene regulated by p53. Cancer Res. 1997; 57 : 3281-7.

43. von der Heide EK, Neumann M, Vosberg S, James AR, Schroeder MP, Ortiz-Tanchez J, Isaakidis K, Schlee C, Luther M, Johrens K, Anagnostopoulos I, Mochmann LH, Nowak D, et al. Molecular alterations in bone marrow mesenchymal stromal cells derived from acute myeloid leukemia patients. Leukemia. 2016. https://doi.org/10.1038/ leu.2016.324.

44. Furukawa T, Kuboki Y, Tanji E, Yoshida S, Hatori T, Yamamoto M, Shibata N, Shimizu K, Kamatani N, Shiratori $\mathrm{K}$. Whole-exome sequencing uncovers frequent GNAS mutations in intraductal papillary mucinous neoplasms of the pancreas. Sci Rep. 2011; 1: 161. https://doi.org/10.1038/ srep00161.

45. Neben K, Tews B, Wrobel G, Hahn M, Kokocinski F, Giesecke C, Krause U, Ho AD, Kramer A, Lichter P. Gene expression patterns in acute myeloid leukemia correlate with centrosome aberrations and numerical chromosome changes. Oncogene. 2004; 23: 2379-84. https://doi. org/10.1038/sj.onc. 1207401 .

46. Wertheim GB, Smith C, Luskin M, Rager A, Figueroa ME, Carroll M, Master SR. Validation of DNA methylation to predict outcome in acute myeloid leukemia by use of xMELP. Clin Chem. 2015; 61: 249-58. https://doi. org/10.1373/clinchem.2014.229781.

47. Yan P, Frankhouser D, Murphy M, Tam HH, Rodriguez B, Curfman J, Trimarchi M, Geyer S, Wu YZ, Whitman SP, Metzeler K, Walker A, Klisovic R, et al. Genome-wide methylation profiling in decitabine-treated patients with 
acute myeloid leukemia. Blood. 2012; 120: 2466-74. https:// doi.org/10.1182/blood-2012-05-429175.

48. Jones PA. Functions of DNA methylation: islands, start sites, gene bodies and beyond. Nat Rev Genet. 2012; 13: 484-92. https://doi.org/10.1038/nrg3230.

49. Costello JF, Fruhwald MC, Smiraglia DJ, Rush LJ, Robertson GP, Gao X, Wright FA, Feramisco JD, Peltomaki P, Lang JC, Schuller DE, Yu L, Bloomfield CD, et al. Aberrant $\mathrm{CpG}$-island methylation has non-random and tumour-type-specific patterns. Nat Genet. 2000; 24: 132-8. https://doi.org/10.1038/72785.
50. Man $\mathrm{CH}$, Fung TK, Wan $\mathrm{H}$, Cher CY, Fan A, Ng N, Ho C, Wan TS, Tanaka T, So CW, Kwong YL, Leung AY. Suppression of SOX7 by DNA methylation and its tumor suppressor function in acute myeloid leukemia. Blood. 2015; 125: 3928-36. https://doi.org/10.1182/ blood-2014-06-580993.

51. Qu Y, Siggens L, Cordeddu L, Gaidzik VI, Karlsson K, Bullinger L, Dohner K, Ekwall K, Lehmann S, Lennartsson A. Cancer-specific changes in DNA methylation reveal aberrant silencing and activation of enhancers in leukemia. Blood. 2017; 129: e13-e25. https://doi.org/10.1182/ blood-2016-07-726877. 